\title{
Legitimidad democrática, Rousseau y Kant. Respuesta a los comentarios de Aldao y Keinert
}

\author{
Democratic Legitimacy, Rousseau and Kant. \\ Reply to the Comments of Aldao and Keinert
}

Jan-R. Sieckmann ${ }^{*}$

Recepción y evaluación de propuesta: 28/05/2016.

Aceptación: 17/04/2016.

Recepción y aceptación final: 22/10/2016.

Resumen: El texto responde a las críticas realizadas por Martín Aldao y Mauricio Keinert al texto "Autonomía moral y legitimidad democrática", presente en esta revista.

Palabras clave: legitimidad democrática, Rousseau, Kant

\begin{abstract}
The article responses to the critics advances by Martín Aldao and Mauricio Keinert to the essay "Moral Autonomy and Democratic Legitimacy", included in this issue.
\end{abstract}

Keywords: democratic legitimacy, Rousseau, Kant

En sus comentarios, Martín Aldao y Mauricio Keinert toman distintos enfoques acerca de mi propuesta sobre autonomía y legitimidad democrática. Aldao desarrolla esta concepción con respecto al tema de la legitimidad democrática. Keinert la critica y propone estudiar a Rousseau y Kant. Aunque no discuten el punto central -la construcción de la autonomía por la concepción de la ponderación de argumentos normati-

* Universidad de Erlangen - Universidad de Buenos Aires.

Correo electrónico: jan.sieckmann@fau.de, jansieckmann@yahoo.de 
vos-, los dos comentarios ofrecen interesantes puntos para discutir. Voy a empezar con Aldao, porque además de sus comentarios resume muy bien mi concepción de la legitimidad democrática.

\section{I.}

Aldao propone una reconstrucción de la tesis de la argumentación autónoma en el marco de una comprensión deliberativa de la democracia y una teoría no positivista del derecho. Además analiza dos procesos político-constitucionales con el objetivo de probar el marco conceptual desarrollado, uno acerca de la función contra-mayoritaria de la justicia en un conflicto norteamericano, el otro acerca de la función democrática de la justicia en el reconocimiento del matrimonio igualitario en la Argentina.

Primero, reconoce que la tesis de la argumentación autónoma permite comprender mejor la conexión entre la teoría de los derechos fundamentales, la teoría de la argumentación jurídica y la teoría deliberativa de la democracia (sec. 1.1). Correctamente entiende la concepción de la argumentación autónoma como un paradigma fuerte de democracia deliberativa: la que entiende la deliberación como condición de posibilidad de la democracia y de este modo conecta el concepto de legitimidad del derecho con la idea de democracia en el seno de la teoría del derecho. Como Aldao destaca, la conexión se sigue de un elemento de la argumentación autónoma que consiste en la reflexión intersubjetiva respecto de las diversas concepciones normativas de los agentes autónomos, considerando la situación de conflicto de las diversas opiniones y evaluando cuál sea la norma adecuada para ésta situación. De esta manera, la tesis de la argumentación autónoma permite ver no sólo la analogía estructural entre la producción de derecho legítimo y la argumentación racional, sino también el modo concreto en que interactúan los principios de autonomía individual y de soberanía popular (sec. 1.1).

Otro punto que destaca Aldao es el principio de universabilidad que se encuentra en la base de la tesis de la argumentación autónoma y que permite trazar una distinción que resulta de la mayor impor- 
Legitimidad democrática, Rousseau y Kant. Respuesta a los comentarios...

tancia cuando se analizan las instituciones democráticas. Desde una comprensión deliberativa, el quid de la democracia no radica en las mayorías, sino en la imparcialidad (sec. 1.2).

Un punto central de mi concepción de la fundamentación de normas es el criterio de la convergencia razonable, lo que permite, si es necesario, sostener que las normas son vinculantes y, en este sentido, válidas objetivamente, incluso para quienes se oponen a las mismas. Aldao destaca dos puntos en que la concepción de la democracia deliberativa requiere convergencia. El primero es una mínima convergencia respecto de (i.) la utilización de la argumentación en lugar de la fuerza como medio de resolución de los conflictos de intereses, (ii.) el diseño de los mecanismos a través de los cuales el procedimiento argumentativo se ve institucionalizado y (iii.) la determinación de una constelación de garantías mínimas comunes para el ejercicio del razonamiento autónomo que podríamos denominar derechos fundamentales. (sec. 1.2., democracia y convergencia). Con razón nota que estos presupuestos no son desarrolladas en mi concepción de la democracia.

Por otro lado, Aldao nota que la democracia puede ayudar a la convergencia, pero no puede construirla desde cero. Para que la democracia funcione no hace falta que todos tengamos los mismos derechos, hace falta que todos tengamos las mismas necesidades, sólo de la comunidad de intereses puede surgir una ley que protegiendo el interés de todos proteja el de cada uno. (sec. 1.2) Sin embargo, esta tesis me parece demasiado fuerte. Aunque la existencia de intereses en común ayuda a reforzar el compromiso de los ciudadanos con el sistema democrático, no parece necesario que todos tengan que tener las mismas necesidades. Alcanzaría, de hecho, con que se respeten mutuamente las necesidades de cada uno, aunque sean diferentes. Con todo, seguramente la existencia de intereses en común ayudaría al funcionamiento de la democracia. De ahí surge la pregunta acerca de en qué medida está justificada, o incluso si resulta necesario tratar de apoyar el desarrollo de intereses en común desde la perspectiva del principio democrático.

Con respecto a la relación entre democracia y derechos fundamentales, en la medida en que la argumentación autónoma consti- 
tuye la base de la legitimidad de los estados democráticos, es posible reconstruir el catálogo de derechos liberales como un primer intento por definir las condiciones de ejercicio del razonamiento autónomo: condiciones materiales (propiedad, industria y libre circulación), condiciones sociales (igualdad formal ante la ley), condiciones culturales (libertad de prensa, de enseñanza y de conciencia). (sec. 1.3) Sin embargo, por ser dependiente de la argumentación, resulta imposible definir de modo sustancial el contenido de los derechos fundamentales; pero sí es posible ver que, estructuralmente, existen una serie de condiciones muy cercanas al núcleo de lo que cada época entiende por autonomía, cuya vulneración por parte del Estado no puede ser tolerada. Aldao reconoce que mientras en una sociedad económica, social y culturalmente homogénea este núcleo de condiciones mínimas es muy sencillo de determinar, en sociedades fracturadas es poco probable que todos acuerden en un límite común. En este sentido la tesis de la argumentación autónoma impone una fuerte carga al Estado en términos de garantía integral de la autonomía de la población, en forma de condiciones de legitimidad de la autoridad de las instituciones políticas, y por ende directamente exigibles. (sec. 1.3)

En el modelo de la argumentación autónoma el poder judicial puede reclamar, entonces, dos funciones de control (sec. 1.4). Por un lado se preserva su rol tradicional de verificar que las decisiones de los poderes públicos se adecuen a lo que la sociedad considera "derechos constitucionales". Pero además el poder judicial puede jugar, dadas ciertas condiciones, un rol democrático en términos de rectificación del proceso de deliberación pública.

Tengo poco para añadir. Aldao presenta muy bien los puntos centrales de mi concepción de la legitimidad democrática, señalando también algunos puntos para desarrollar.

\section{II.}

Keinert toma una posición más crítica. Una primera crítica afirma que existe una ambigüedad estructural en mi análisis del concepto de autonomía. Según Keinert, 
Legitimidad democrática, Rousseau y Kant. Respuesta a los comentarios...

... si comenzamos la lectura del artículo teniendo en cuenta una idea de autonomía cuya característica principal sólo se refiere a los intereses propios de un individuo, al final de este mismo artículo el concepto de autonomía tiene en cuenta el concepto de intersubjetividad, de posibles criterios universalistas y la consideración de buenos argumentos. (sec. 1).

Así, Keinert da la impresión de que hay un cambio en mi concepción de autonomía. De hecho, sin embargo, empiezo con el tema de la legitimidad política (sec. I), sigo con la necesidad de buscar consentimiento de los que se encuentran sujetos a estas decisiones políticas (sec. II) y de ahí llego a la discusión de la concepción de autonomía (sec. III). Como conclusión, ofrezco distintas definiciones de autonomía en sentido estructural, individual y político (sec. III.3). Así, trato de reconstruir y precisar la idea de la autonomía. En ningún momento adopto la posición de que la autonomía se refiere sólo a los intereses propios de un individuo.

Otra crítica de Keinert es la supuesta identificación del concepto de autonomía con el de autonomía individual. Sin embargo, como distingo explícitamente entre concepciones de autonomía estructural, individual y política, la tesis que identifica autonomía con autonomía individual es obviamente incorrecta.

Otro punto en que Keinert se equivoca es que mi concepción sea "una visión extremadamente liberal del concepto de individuo, visto de manera inflexible como portador de intereses determinados de forma puramente mecánica". No sé de dónde Keinert saca esa idea. No se encuentra en mi texto, y Keinert no da ninguna referencia concreta dentro del mismo.

También se equivoca Keinert cuando alega que "es en estos términos [creo que Keinert se refiere con términos a intereses] que la paradoja de la autonomía nos es presentada". Sin embargo, la paradoja de la autonomía está introducida como una contraposición de la aceptación individual de normas que debe ser por un lado libre y por otro lado vinculada por normas. No está presentada en términos de intereses. La aceptación individual no depende directamente de intereses individuales, sino de sus juicios normativos. 
Además, Keinert no distingue entre razones e intereses. Cuando cita mi tesis: "Pero si alguien pudiera con razón rechazar el poder político, no existiría una justificación o legitimidad de este poder", la interpreta como tesis de que de hecho es el individuo y, por lo tanto, su voluntad y sus intereses, el principal criterio de legitimidad del poder político y no exactamente el concepto de autonomía. Así, identifica razones para rechazar el poder político con voluntad e intereses de los individuos, lo que es equivocado y de ninguna manera es lo que sostengo en mi texto.

En el mismo sentido, Keinert se refiere a mi tesis de que la idea del consentimiento de cada uno de los individuos en relación al poder será el "núcleo de la legitimidad democrática" y alega que "una vez más es evidente que el criterio de apreciación de Sieckmann se refiere a la voluntad misma del individuo más que precisamente al concepto de autonomía". Pero el consentimiento que, en mi concepción, presupone la legitimidad democrática, no es lo mismo que la voluntad individual sin reflexión racional e intersubjetiva.

En fin, Keinert constata que la idea de que cada uno de los individuos dará su consentimiento de acuerdo con sus propios intereses no parece armonizar con los conceptos de autonomía tal como lo encontramos en Rousseau y, principalmente, en Kant. Tampoco armoniza con mi concepción de autonomía, al menos si se entiende intereses como algo irreflexivo y no como el resultado de un proceso de deliberación racional e intersubjetiva.

A continuación, Keinert defiende la tesis de que aún cuando la filosofía política moderna haya partido de la cuestión del individuo, esto no la convierte necesariamente en liberal. El problema que Keinert encuentra en mi artículo se refiere "a la reciprocidad entre el concepto de autonomía moral y el de autonomía individual, presupuesta por el artículo, pero que, sin embargo, no es abordada de forma directa" (secc. 2). Como Keinert presenta mi concepción de autonomía individual de manera distorsionada, es difícil discutir su exposición como comentario a mi texto. Sin embargo, queda un punto para discutir. Para mí, la legitimidad política depende de juicios normativos de los individuos sujetos al poder político. Estos juicios se encuentran 
Legitimidad democrática, Rousseau y Kant. Respuesta a los comentarios...

relacionados con los intereses de los individuos, los que deben ser legítimos, integrados en un "plan de vida" coherente, balanceados con intereses divergentes y sujetos a un proceso de reflexión intersubjetiva. La pregunta es si en Rousseau o Kant se encuentra una plausible alternativa para una concepción de la legitimidad política.

Como explica Keinert, Rousseau plantea el problema de la relación entre el individuo y la asociación de individuos, que se convertirá en un cuerpo político, como "el pasaje de la libertad natural a la libertad convencional de estos últimos. Tal pasaje le permitirá afirmar que cada uno, uniéndose a todos, no obedece sino a sí mismo, pues, al formar parte ahora del cuerpo político soberano, el individuo, en tanto ciudadano, al obedecer la voluntad general, se obedece a sí mismo. Esto significa, y es lo importante, que cada uno cede su libertad natural, y sus derechos naturales, en favor de la unidad del cuerpo político, volviendo a todos, desde el punto de vista moral y politico, ciudadanos autónomos y, por lo tanto, iguales y libres desde el punto de vista de la convención alcanzada por el pacto social". (secc.2)

Esta concepción tiene dos defectos. En el plano conceptual, confunde autonomía con el conjunto de, por un lado, formar parte de un cuerpo político participando en procedimientos políticos, y, por otro lado, estar sujeto al poder político. Como explica Keinert, para Rousseau "una doble relación nos permite pensar el concepto de autonomía: en primer lugar, él se refiere a la unidad de la asociación que es soberana y promulga las leyes del Estado, la forma institucional de esta soberanía; en segundo lugar, los ciudadanos como partícipes de esta soberanía son agentes de estas leyes, pero, por ser sujetos y pasivos, están también sometidos a ellas". (secc. 2) Pero formar parte de un cuerpo político y participar en sus procedimientos no significa que alguien legisle sobre sí mismo. Podría ser que una minoría estructural participase en procedimientos políticos de manera libre e igual, pero nunca tenga la chance de que sus demandas sean reconocidas. Siempre pierden contra los votos de la mayoría. Describir eso como auto-legislación parece poco plausible. Entonces el ciudadano individual no puede ser considerado como autónomo. Por otro lado, el cuerpo político tampoco es autónomo en el sentido literal, porque no 
legisla sobre sí mismo sino sobre los ciudadanos. No encontramos una concepción de autonomía plausible en Rousseau.

Otro defecto de la teoría de Rousseau se encuentra en el plano justificatorio. Podría preguntarse por qué un individuo debe ceder su libertad natural y asociarse con otros en un cuerpo político, y con quiénes y en qué cuerpo político. Si no se justifica el pasaje de la libertad natural a la libertad convencional en términos de los intereses, demandas y juicios de los individuos mismos, no hay justificación que corresponda a la idea de la autonomía.

Parece que en Kant se resuelve este problema justificatorio. Como Keinert explica, Kant da al acto de asociación un significado a priori y trascendental (secc. 2). Pero su concepción de autonomía tampoco resulta satisfactoria.

Respecto de la autonomía moral, Keinert caracteriza la concepción de Kant de la siguiente manera: "Si pensáramos la representación de la ley por medio del concepto de deliberación, podemos afirmar entonces que al comparar la máxima con la ley, la razón inmediatamente se pregunta por la universalidad de la forma de la máxima, y tal universalidad, cuando es positiva, supone el hecho de que todos los otros seres racionales (exigencia de totalidad de la razón) prestarían su consentimiento a tal acción. En este sentido, al representar la ley y actuar por medio de esta paralegalidad (legiformidade), el individuo delibera en el interior de un todo moral que, a su vez, se confundirá con el valor moral incondicional de su acción, pues es él el que dará legitimidad a la regla de la acción. Es en este movimiento de representación (o de deliberación) que podemos reconocer el concepto kantiano de autonomía, pues al representar la ley, al hacer la comparación de la máxima con la ley, este individuo legisla por medio de su actividad racional para sí mismo y para todos los otros seres racionales, colocándose de este modo en la posición de legislador y de destinatario de la ley." Podemos constatar que determinar el valor moral de la acción requiere un acto de voluntad del agente quien formula la máxima comparada con la ley moral. Además, este acto formula una ley que pretende ser válida para todos los otros seres racionales. Sin embargo, no es claro qué significa "pretende" en este contexto. Como la au- 
Legitimidad democrática, Rousseau y Kant. Respuesta a los comentarios...

tonomía individual permite que cada agente autónomo desarrolle su propia posición moral, la pretensión de validez no se puede entender en el sentido de que una ley establecida por un agente autónomo sea válida para todos los otros seres racionales. Cuando la ley moral no determina una única máxima que puede ser considerada válida para todos, existen diversas opciones. Entonces, la pregunta es si se puede reclamar que una de esas máximas será una norma vinculante. Esto es justamente el tema que estoy tratando. Mi respuesta es que los individuos pueden sostener que los demás deben reconocer tal norma como válida, pero no pueden constatar que es válida en el sentido que sea vinculante para todos los demás.

Entonces, existe cierto paralelo entre la autonomía moral tal como al presenta Kant y la autonomía individual en mi concepción de autonomía. Ambas tratan de explicar cómo se puede explicar la idea de la validez de las normas en sentido moral. Las máximas que se corresponden con la ley moral determinan cuándo una acción tiene valor moral. Pero cuando la ley moral no determina sólo una máxima única, diversas máximas son posibles. Cada agente autónomo puede definir por sí mismo cuáles acciones tengan valor moral para el (es decir, valor moral positivo). Para mí, éste es el tema de la autonomía individual. Cada individuo llega a juicios normativos y reclama que ciertas normas deben ser reconocidas como válidas, pero no puede reclamar que estas normas sean vinculantes para todos los demás, porque otros agentes pueden juzgar de manera distinta. En comparación con Kant, mi concepción se encuentra más estructurada. Distingue entre, primero, la formación de argumentos normativos, basados en intereses y demandas de los agentes autónomos. Estos deben ser legítimos en el sentido que no se dirigen contra la autonomía de otros agentes. Deben tener universabilidad en el sentido de que cada agente autónomo puede reconocerlo como demanda. Segundo, sigue la ponderación de los argumentos normativos en cuanto que se encuentren en conflicto. De nuevo, los resultados deben ser aceptables para cualquier agente autónomo. Tercero, es necesario una reflexión intersubjetiva acerca de la pregunta sobre cuál norma debe ser reconocida en una situación de posiciones normativas divergentes. De nuevo, rige una exigencia de 
universabilidad, porque se debe tomar en cuenta la posición de cada agente autónomo y preguntarse cómo sería una norma que todos pudieron aceptar como vinculante. Así, se define de manera más precisa lo que significa formular una máxima que está sujeta a la ley moral en la interpretación como principio de universabilidad. Además, la estructura de los argumentos normativos explica cómo los agentes autónomos pueden reclamar que una norma sea reconocida como vinculante, sin constatar que esta norma de hecho es vinculante para todos. Este reclamo normativo es posible aunque no solo una única posición normativa sea posible. En cambio, la comparación de máximas con la ley moral no explica porque alguien sostiene una norma como moralmente válida frente a otra norma que sería igualmente posible.

Queda el tema de la vinculatoriedad de las normas morales. Keinert trata este tema en relación con la autonomía política. Explica que "desde un punto de vista universal, el ciudadano debe hacer uso público de su razón, cuestionando las mismas normas, y que esto establece una relación entre ciudadanos, en que la obediencia tiene como condición de posibilidad el libre pensar. Es porque hago uso público de mi razón que obedezco las leyes constitucionales, por ejemplo". Para Keinert, hacer uso de la razón pública tiene en cuenta algunas características importantes del concepto de autonomía (secc. 3). Sin embargo, el uso público de la razón o la deliberación pública, es decir, reflexionar y crítica las normas, no hace que esas normas sean vinculantes. Puede ser una condición necesaria, pero seguramente no suficiente.

Según Keinert, lo que daría legitimidad al proceso de decisión sería la posibilidad de todos los implicados que hicieren uso público de su razón, es decir, deliberasen (secc. 5). No tengo ninguna objeción contra eso como condición necesaria de la legitimidad política. Pero no es suficiente para explicar como se puede considerar normas como vinculante para todos en una moral basada en la autonomía. En cambio, mi artículo trata de desarrollar una concepción de argumentación (o deliberación) autónoma, que analiza si y en qué sentido se puede reclamar la validez de normas como vinculantes frente a agentes autónomos. Esta concepción está basada en una concepción de autonomía individual, empezando con argumentos normativos constituidos por 
Legitimidad democrática, Rousseau y Kant. Respuesta a los comentarios...

demandas de agentes autónomos, las que deben ser legítimas según un criterio de universabilidad, sigue con la ponderación de estos argumentos y la reflexión intersubjetiva acerca de posiciones normativas divergentes, y por último explica el criterio de convergencia razonable como condición que permite reclamar la vinculatoriedad de normas incluso a agentes autónomos que siguen sosteniendo una posición normativa opuesta. No veo nada parecido en Kant o en la reconstrucción de Kant que Keinert ofrece.

En fin, me parece demasiado fuerte la tesis de Keinert de mi manera de presentar el problema de la autonomía como paradoja no tenga sentido en el marco conceptual kantiano (secc. 4). Como los agentes autónomos deben formular máximas que son comparadas con la ley moral, existe la posibilidad de que los diversos agentes formulen diversas máximas incompatibles entre sí pero cada una compatible con la ley moral. Así tenemos el problema de, por un lado, que las normas morales deben depender de los juicios morales de los agentes autónomos, los que son libres en el marco de la ley moral, y, por otro lado, que ellos mismos deben estar obligados por las normas morales. Pero, si son libre de eligir una norma u otra, ¿cómo pueden entenderse como vinculado por la norma eligida? Creo que se puede entender este problema también en el marco de la concepción kantiana. Solo que no se puede dar una respuesta adecuada.

Para concluir, me parece que, aunque Rousseau y Kant ciertamente tienen sus méritos históricos, sus teorías no son tan adecuadas para entender la idea de autonomía como autolegislación, y que adherirse demasiado a las "autoridades filosóficas" puede impedir analizar los problemas de la autonomía. 